\title{
Operative Fixation of Radial Head Fractures
}

\author{
John T. Capo, MD and Dan Dziadosz, MD \\ Division of Hand and Microvascular Surgery \\ Department of Orthopaedics \\ New Jersey Medical School \\ Newark, NJ
}

\section{ABSTRACT}

The radial head is an important structure that is crucial to the stability of both the elbow and forearm. Fractures of the radial head occur commonly and often are displaced or cause impingement to forearm rotation. Recent studies have further demonstrated the importance of maintaining or reconstructing the rigid buttress of the radial head. Advances in implant technology have benefited orthopedic surgeons in their attempts at fixation of the radial head and also in rigid implant arthroplasty. Partial articular fractures of the radial head are relatively simple to repair and can be stabilized with headless or buried screws. A complete articular fracture is more challenging and requires the use of rigid, lowprofile, and, often, fixed-angle plates placed in the nonarticular portion of the radial head. Associated ligament tears in the elbow magnify the importance of restoring the stabilizing effect of the radial head. Radial head excision should rarely be done and only after a thorough examination of the ligamentous structures about the elbow and forearm demonstrate complete stability.

Keywords: radial head, fractures, ORIF, stability

\section{HISTORICAL PERSPECTIVE}

Historically, treatment of radial head fractures primarily involved excision of the fracture fragments as well as the remaining pieces of the intact radial head. This treatment potentially created scenarios of instability, motion deficits, proximal migration of the radius, and distal radioulnar joint (DRUJ) dysfunction. ${ }^{1,2}$ With the development of advanced techniques in open-reduction and internal fixation (ORIF), the radial head can often be rigidly fixed and the stability of the elbow can be maintained. ${ }^{3}$

Several comparisons among ORIF, prosthetic arthroplasty, excision, and nonoperative treatment have been investigated. $^{4-8}$ It appears that the trend for the treatment of significantly displaced radial head fractures is for stable fixation with plates and/or screws or re-

Reprints: John T. Capo, MD, 90 Bergen St, DOC 1200, Newark, NJ 07103 (e-mail: capojt@umdnj.edu).

placement with rigid implant arthroplasty. Beingessner et $\mathrm{al}^{9}$ investigated the biomechanical effects of radial head excision and prosthetic arthroplasty on elbow kinematics in a cadaver model. They demonstrated that replacement of the radial head with a metallic implant improves stability but does not return the elbow to a normal condition. This effect was most clearly seen with associated ligamentous instability and emphasized that rigid implants do not exactly reproduce the dynamics of the intact or rigidly fixed radial head. The native radial head is also elliptical and sits at approximately a 15-degree angle to the radial shaft. These anatomic characteristics are not replicated by implant arthroplasty. For these and other reasons, preservation of the native radial head is desirable, particularly in younger high-demand patients.

\section{FRACTURE CLASSIFICATION}

One of the common systems for classifying radial head fractures is that of Mason, ${ }^{10}$ originally described in the 1950s. This system separated these fractures into 3 types. Type I fractures were described as nondisplaced fractures that were treated most successfully by closed (nonoperative) means. Type II fractures were displaced fractures involving only a portion of the radial head, with some remaining head continuous with the radial shaft or minimally displaced neck fractures. Type III fractures were comminuted fractures involving the entire radial head whose treatment options were historically limited to excision.

Since the time of the original classification system several modifications have been made. One of the firsts was by Morrey in the 1980s. This modification included a quantitative description of fracture displacement (a fragment involving $30 \%$ or more of the articular surface and $2 \mathrm{~mm}$ or more of displacement was deemed unacceptable) and fracture dislocations of the elbow. ${ }^{11}$

One of the more recent modifications, made by Hotchkiss, ${ }^{5}$ appears to help with treatment decision making. Type I fractures are characterized by minimal displacement, with forearm rotation limited by acute pain and swelling, intraarticular displacement of less 
than $2 \mathrm{~mm}$, or a marginal lip fracture. Type II fractures are displaced fractures with more than $2 \mathrm{~mm}$ of displacement of the head or neck, usually with blocked or incongruous motion. There is minimal comminution, but there is more than just a simple marginal lip fracture of the radial head. These fractures are usually amenable to ORIF. Type III fractures are severely comminuted. These are irreparable based on radiographic or intraoperative appearance, and excision or replacement is usually required to allow motion.

\section{OPERATIVE INDICATIONS}

The decision to perform ORIF of radial head fractures involves several factors. The character of the radial head fracture must be analyzed as well as associated ligamentous and bony injuries around the elbow. This may require advanced preoperative diagnostic studies including specialized radiographs, computed tomography scans, and magnetic resonance imaging. A helpful radiograph is the oblique radiocapitellar, or Coyle, view that shows the radial head in profile without the overlying coronoid. Plain films alone have been shown to be inaccurate in estimation of the fracture size and displacement. ${ }^{12}$ In our opinion, radiographic indications for operative treatment of radial head fractures are neck fracture with greater than 15 degrees of angulation or greater than $25 \%$ displacement, intra-articular fractures greater than $25 \%$ of the radial head with more than 2 $\mathrm{mm}$ of articular step-off or that create a mechanical block to motion. Although this is our recommendation, intra-articular displacement greater than $2 \mathrm{~mm}$ has been shown in some long-term studies to not preclude a good clinical result. ${ }^{13}$ Other scenarios that would make operative stabilization necessary include longitudinal radial instability seen in an Essex-Lopressti injury or elbow varus-valgus laxity that may be caused by concomitant collateral ligament injury.

Patient variables that should be factored into the surgical decision making process include age, hand dominance, occupation, as well as the physical demand placed upon the elbow. Operative fixation should be offered, when indicated, to all patients who can medically tolerate the operative procedure. Excision without replacement in a higher demand individual might lead to proximal migration of the radius with decreased function at both the elbow and the wrist. ${ }^{14}$

\section{CONTRAINDICATIONS}

Factors that preclude operative intervention are related to concomitant injuries, soft tissue integrity, local healing environment, generalized patient health, as well as patient demand. In the setting of associated severe fracture dislocation of the elbow or comminuted forearm fractures, the long-term result of radial head repair, especially with regard to restoration of forearm rotation, may be compromised. ${ }^{15}$ In this situation, another method of fixation might be necessary to stabilize the elbow, such as hinged external fixation. ${ }^{16}$ If severe soft tissue damage exists (ie, burns, soft tissue loss, or infection), operative intervention should be delayed for adequate soft tissue healing. Patient factors will also dictate the timing and need for surgery. Should the patient be too medically unstable to undergo operative intervention, then surgery must be delayed. Finally, if the radial head is excessively comminuted and unreconstructable, then implant arthroplasty may be more appropriate.

\section{PREOPERATIVE PLANNING}

The elbow should be examined for tenderness, neurovascular status, soft-tissue condition, and range of motion (ROM). Tenderness or ecchymosis at the medial joint line may represent a medial collateral ligament injury, whereas tenderness at the DRUJ could reflect longitudinal instability of the elbow. All 3 major nerves in the arm are examined, with particular attention to the posterior interosseous nerve (PIN). Forearm rotation is assessed to see if a mechanical block to motion exists. If pain is excessive at the elbow, aspiration of the joint hematoma followed by injection of a local anesthetic is helpful in assessing motion and relieving pain.

Associated injuries that are critical include coronoid fractures, medial and lateral collateral ligament (LCL) injuries, and DRUJ injuries. If there was an associated dislocation, then there is often an associated, seemingly insignificant, coronoid process fracture. Repair of this coronoid fracture will often provide increased stability to the elbow and allow earlier ROM. With an incompetent coronoid, the resistance to anteroposterior (AP) instability and tendency for resubluxation is increased, and the need for a stable radial head is further magnified. Damage to the interosseous ligament of the forearm and DRUJ can induce proximal migration of the radius with an absent or incompetent radial head. ${ }^{14}$

Equipment required should include all that is needed for excision, internal fixation, or replacement arthroplasty. Small screws ranging from 1.3 to $2.4 \mathrm{~mm}$, as well as headless compression screws are often useful for head fragments. Some type of small plating system, either a general system or a dedicated radial head system, should be available in the operating room. Finally, a rigid implant arthroplasty of a suitable bearing surface, preferable $\mathrm{Cr}-\mathrm{Co}$, and with sufficient modularity is needed. A large fluoroscopic machine, which allows a wide view of the entire forearm and elbow, is preferable to the mini units. 


\section{INTRAOPERATIVE ASSESSMENT}

The elbow should be carefully and systematically examined in the operating room before the decision for radial head excision versus fixation is made. Each elbow is examined in the following manner. Axial migration is tested with the elbow stabilized on the hand table, the forearm in neutral rotation, and load placed by the surgeon on the fisted hand. Proximal migration of the radius can be appreciated if the radial shaft migrates into the capitellum. Also, the radial shaft can be grasped with a tenaculum and pulled proximally and radial shortening can be observed at the DRUJ on fluoroscopy. Medial collateral ligament integrity should be evaluated with the forearm in pronation and the elbow flexed to 30 degrees. The degree of opening, the feel of the end point, and radiographic appearance are all observed with this stress maneuver. Radial head incompetence will exacerbate posterior lateral instability, which is tested in elbow supination, valgus, and axial load. Finally, AP stability should be evaluated in progressive extension. If posterior subluxation of the elbow is seen at more than 30 degrees of flexion, then the radial head should be repaired or replaced. Also, if a complex proximal ulna or coronoid fragment undergoes ORIF, reconstitution of the radial head buttress will help unload the ulna to allow healing.

\section{TECHNIQUE}

\section{Surgical Exposures}

Surgical approaches to the radial head include the classic posterolateral (Kocher) approach between the anconeus and extensor carpi ulnaris (ECU) and a more extensile direct lateral approach. This extensile approach begins at the Kocher interval but distally extends to split the extensor muscles, thus allowing access to the radial shaft.

The Kocher approach starts with an oblique incision beginning over the posterior surface of the lateral epicondyle and continuing downward and distally to a point over the posterior border of the ulna, about $6 \mathrm{~cm}$ distal to the tip of the olecranon. The internervous plane lies between the anconeus, supplied by the radial nerve proper, and the ECU, supplied by the PIN. Full pronation of the forearm moves the PIN away from the operative field. ${ }^{17,18}$ The capsule of the elbow joint is incised longitudinally at the annular ligament to reveal the underlying capitellum and the radial head. The capsule should not be dissected too far anteriorly as the PIN runs over the front of the anterolateral portion of the elbow capsule. Because of the posterior location of this approach, the LCL complex is in danger and care must be taken not to release the origin or insertion of this ligament. The exposure is adequate for access to the radial head alone but is not ideal for exposure to the shaft, because it is difficult to extend distally. ${ }^{19}$

The extensile lateral approach is made over the radial head and neck but anterior to the LCL complex. The skin incision is centered over the lateral epicondyle and extends distally over the radial head and neck, anterior to the anconeus (Fig. 1). The deep muscular interval runs from the lateral epicondyle through the posterior aspect of the ECU muscle attachment proximally. It is critical to stay anterior to the posterior border of the ECU, because it has been shown that these fibers are integral to the LCL complex. ${ }^{20}$ The dissection is carried distally between the ECU and extensor digitorum communis. The muscle fibers are spread and retracted as the anterior portion of the extensor mass is reflected. A useful landmark to look for at this point is the capitellum, and ensure dissection should be anterior to its equator (Fig. 2). As the extensor fibers are spread, the crossing fibers of the supinator will next be seen. These fibers run obliquely at approximately a 45-degree angle from the muscle fibers of the extensor mass (Fig. 3). For better visualization, the anterior extensor sleeve can be released from the epicondyle and repaired later. The posterior extensor mass cannot be safely released because it covers and includes the attachments of the LCL. The supinator can be safely divided for a distance of approximately $3 \mathrm{~cm}$ from the articular surface of the radial head with the forearm in pronation without injuring the PIN. Further distal dissection requires identification of the PIN. The

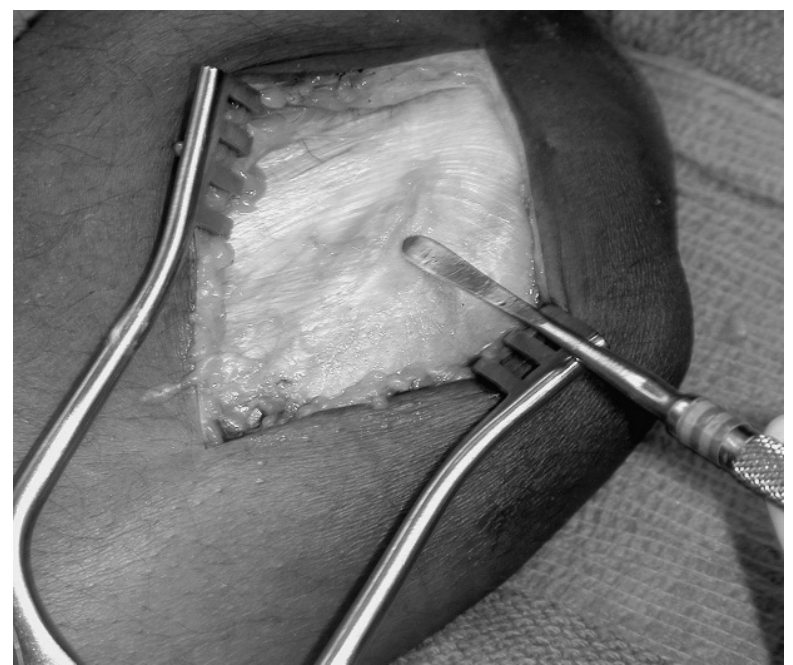

FIGURE 1. The incision is centered over the lateral epicondyle and extends distally over the radial head and neck. There is a raphe seen between the anterior extensor mass and the ECU that can be used as a guide for the deep dissection. 


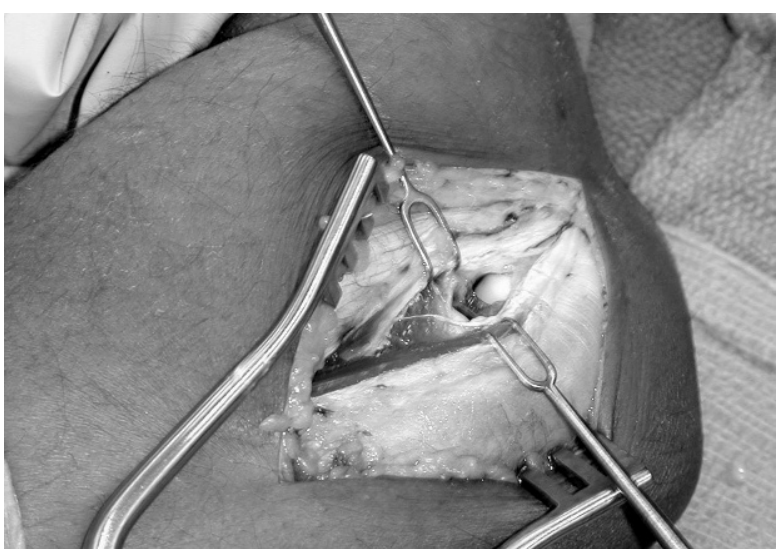

FIGURE 2. As the anterior extensor mass is elevated from the lateral epicondyle and deep capsule, the capitellum is next seen. The deep dissection should stay anterior to the capitellum and, thus, the radial head.

goal of this approach is to protect the posterolateral ligamentous complex and PIN and still provide adequate exposure for fixation.

If the posterolateral incision needs to be extended distally, pronation of the forearm is helpful for protection of the PIN, but it does not completely remove the danger. A cadaveric study performed by Diliberti et al ${ }^{17}$ showed that pronation of the forearm allows exposure of at least the proximal $38 \mathrm{~mm}$ of the lateral aspect of the radius. Additionally, a study performed by Witt and Kamineni $^{21}$ showed that at the distal end of the exposure, the first branches of the PIN are at risk, as those to the ECU, were on average $6.0 \pm 1.0 \mathrm{~cm}$ (range, $4.0-8.4 \mathrm{~cm}$ ) from the articular surface of the radial head.

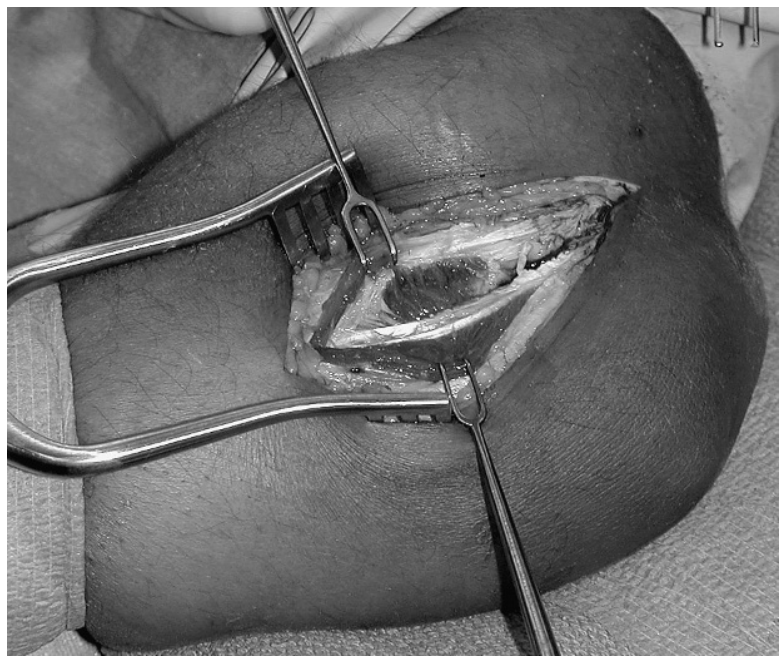

FIGURE 3. The extensor fascia and distal muscle fibers are spread and elevated as a sleeve. Distally and deep, the crossing fibers of the supinator will be seen running obliquely.

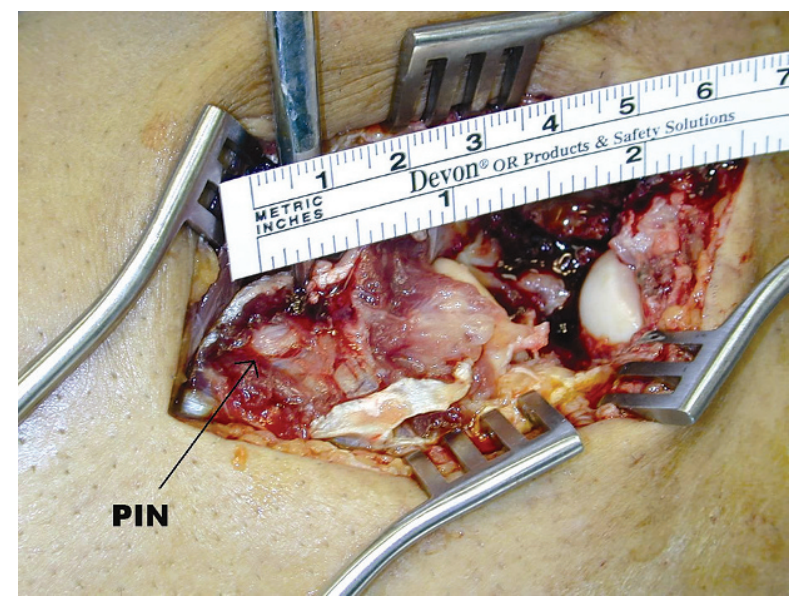

FIGURE 4. The PIN is found anteriorly as it enters the supinator muscle belly at approximately $4.5 \mathrm{~cm}$ from the capitellum and $3.0 \mathrm{~cm}$ from the radial head articular surface.

If a plate is required and more distal dissection is necessary, it is prudent to identify and protect the PIN. The nerve is found anteriorly as it enters the proximal supinator (Fig. 4). It can be dissected through the
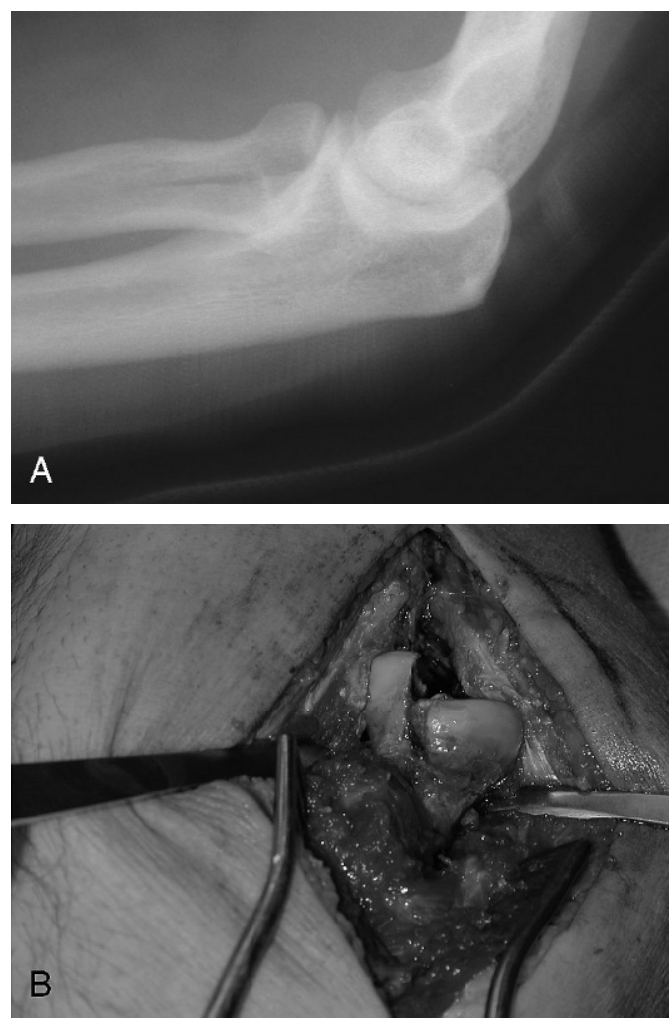

FIGURE 5. A, A lateral radiograph of partial articular fracture of the radial head showing a portion of the head intact to the shaft. B, Clinical photograph demonstrating more articular step-off than appreciated radiographically. 

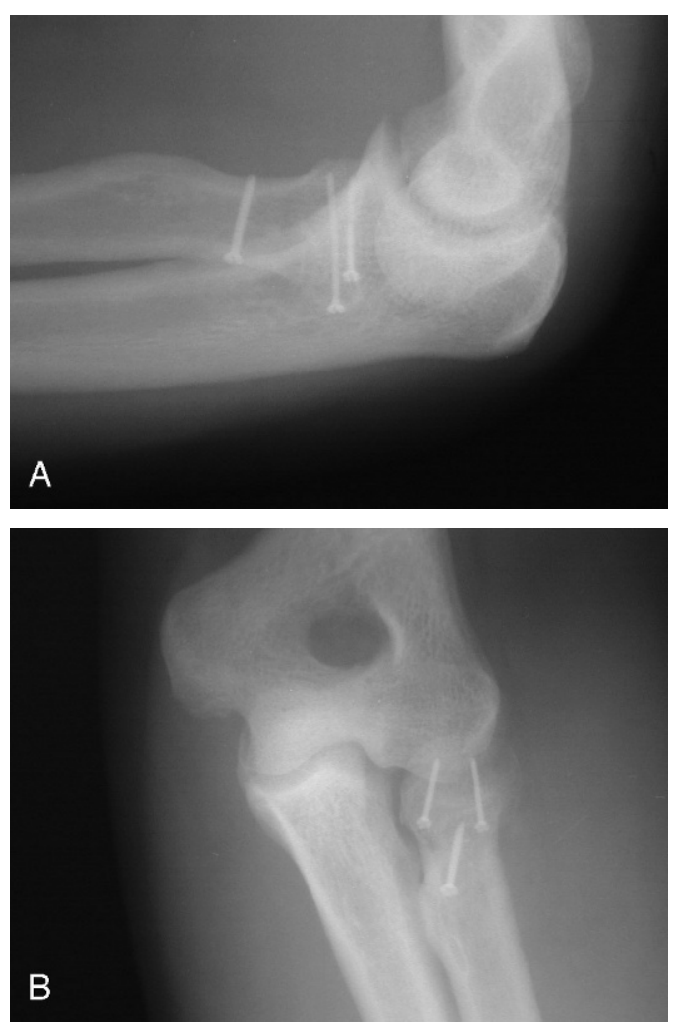

FIGURE 6. Lateral (A) and AP postoperative radiographs (B) demonstrating anatomic fixation of the radial head fracture. A 2.0-mm screw was placed in the neck, whereas two 1.5-mm screws were used to stabilize the radial head.

supinator, or alternatively, the posterior aspect of the supinator can be released and reflected anteriorly while observing the location of the nerve.

\section{Fixation Techniques}

Once the radial head has been adequately exposed through one of the aforementioned approaches, the head fragments need to be fixed together, and then, if necessary, the head is fixed to the shaft. The method of fixation varies greatly and can range from Kirschner (K) wires and headless screws to plates and intramedullary implants. All fixation needs to stay in the nonarticular "safe zone" of the radius so rotation is not limited. This zone comprises approximately 100 degrees and is on the dorsal aspect of the radius, in line with the Lister tubercle of the wrist. With the forearm in neutral rotation, the direct lateral surface of the radial neck is the center of the safe zone. If the boundaries of the safe zone are not violated, then plates and screws can be used without fear of causing impingement of the proximal radioulnar joint. ${ }^{5,22}$ The varying types of hardware used for fixation include crossed $\mathrm{K}$ wires, $\mathrm{T}$ plates, locked plates, and headless screws. ${ }^{15,23,25}$ Provisional fixation of the articular fragments is achieved using small (0.028- and 0.035-in) $\mathrm{K}$ wires.

Partial Articular Fragments. Once provisional fixation has been achieved, definitive fixation is performed using screws to compress and rigidly fix the fragments. This can be done with either headless or lowprofile small headed screws. Screws are either placed in the non-articular portion in standard fashion, or buried well below the surface if placed in cartilage. If the fracture is only a portion of the head, then the fixation can be done in situ (Fig. 5). With a portion of the radial head still continuous with the radial shaft, these fractures are relatively straightforward. A Kocher approach is usually adequate because no distal dissection is
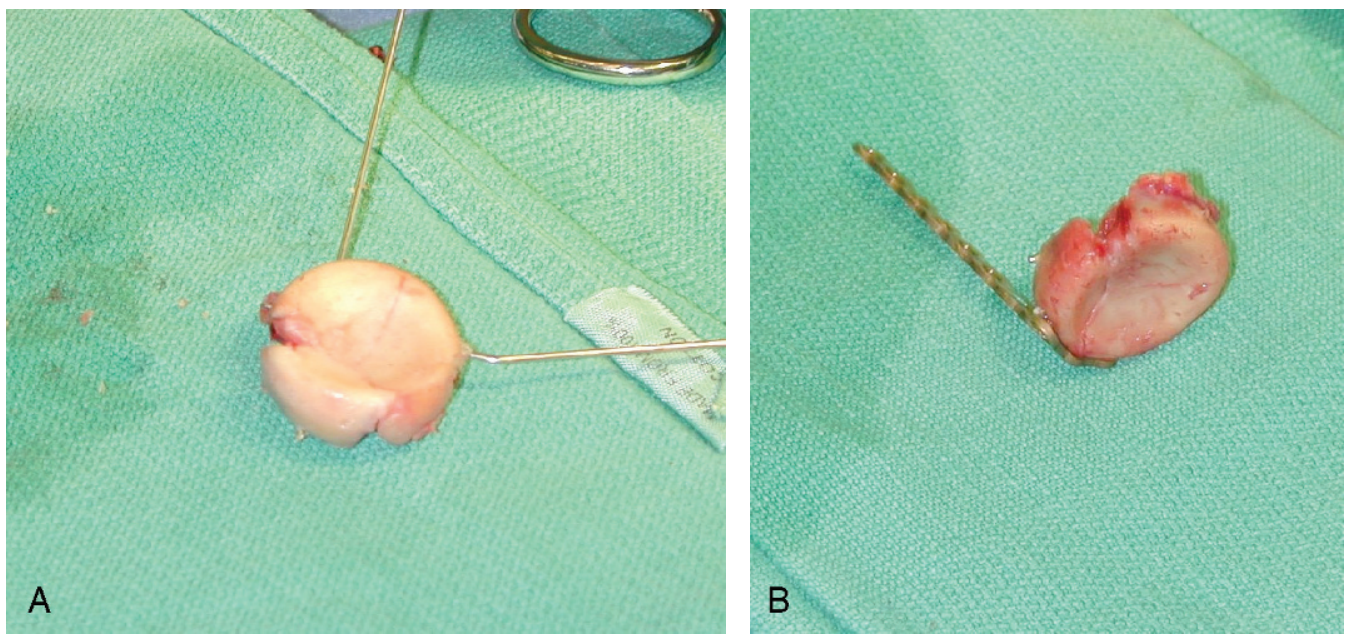

FIGURE 7. A, Multiple articular fragments of radial head are first provisionally fixed with $\mathrm{K}$ wires and buried screws. B, The assembled head is then attached to an appropriate $\mathrm{T}$ plate. 


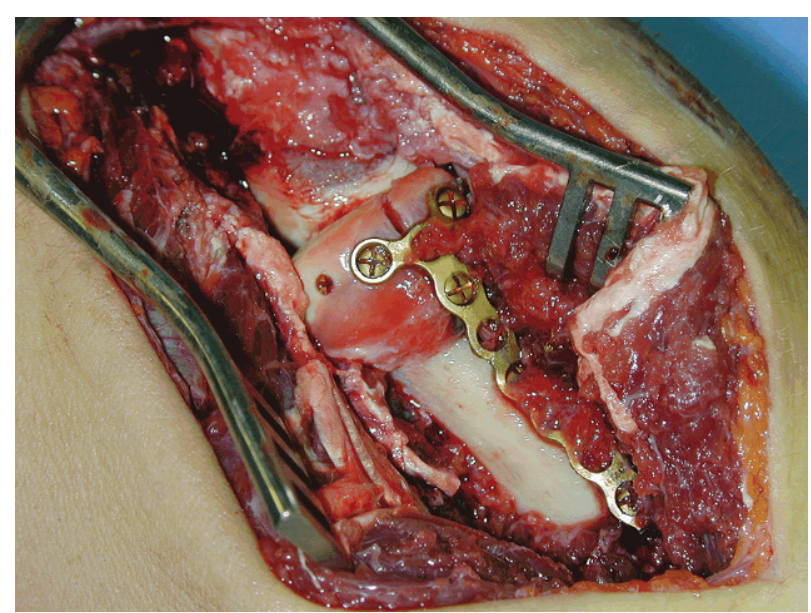

FIGURE 8. The $T$ plate and head construct are next attached to the radial shaft. The plate needs to be contoured to fit to the radial neck. A buried headed screw can be seen outside the plate.

required. The LCL still needs to be protected, and the dissection should stay anterior to the equator of the radiocapitellar joint. Headed screws of 1.3 to $2.0 \mathrm{~mm}$ in diameter may be placed in a standard fashion in the safe zone or should be countersunk if inserted into an articular portion of the radial head (Fig. 6). Care must be taken not to overpenetrate these screws past the far cortex, or joint impingement may occur at the proximal radioulnar joint.

Combined Radial Head and Neck Fractures. If there exists a complete articular fracture, it may be necessary to remove the entire head and fix the head on the back table. With the head free, it can be difficult to tell where the safe zone is; however, careful inspection can demonstrate the safe zone by its absence of cartilage. The head fragments can be assembled to each other with small $\mathrm{K}$ wires and headless screws. The head is next fixed to an appropriate plate, and the head-plate construct is brought into the operative field (Fig. 7). The head should be compressed to the shaft through an eccentric hole in the plate and then with an interfragmentary screw if possible. Secondary to the normal angulation of the radial head on the shaft the plate may lie obliquely when appropriately placed (Fig. 8). Bone grafting is sometimes necessary as well as the use of an additional plate. ${ }^{6}$ Biomechanical investigations have demonstrated that the addition of a lag screw across the radial neck significantly improves stability of the construct in both torsion and bending. ${ }^{24}$

Once adequate reduction and appropriate fixation has been achieved, appropriate soft tissue repair and closure must be performed. The LCL may have been torn at injury, released during the exposure, or weakened by the retraction necessary during fixation. Repair of the LCL can be performed using either suture anchors or transosseus drill holes, with heavy (no. 2 or no. 5) nonabsorbable sutures (Fig. 9). Repair of the LCL will also help tighten the annular ligament, and thus, a separate annular ligament reconstruction is usually unnecessary. ${ }^{5}$ The forearm should now be taken through a full ROM to ensure there is no impingement and that the elbow is stable.

\section{RESULTS}

Recent studies comparing ORIF to alternative treatments have shown that fixation provides superior outcome in terms of function to that of resection alone. Ikeda et $\mathrm{al}^{6}$ compared resection versus ORIF in Mason type II and III radial head fractures and showed that those in the ORIF group had significantly better Broberg and Morrey functional rating scores and ASES Elbow Assessment scores. In addition, King and colleagues ${ }^{25}$ demonstrated that ORIF of these fractures have good outcomes if stable fixation is achieved that permits early ROM.

Ring et $\mathrm{al}^{15}$ performed a retrospective review looking at the functional results after ORIF of fractures of the radial head to determine which fracture patterns were most amenable to this treatment. Their conclusions
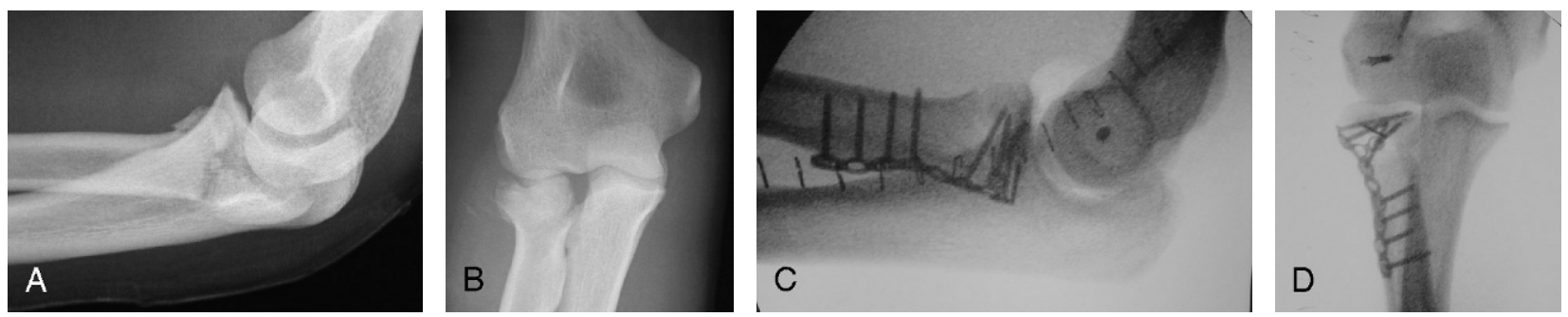

FIGURE 9. Lateral (A) and AP radiographs (B) of a complete articular fracture with significant fragment displacement. Postoperative lateral (C) and AP films (D) showing near anatomic restoration of the radial head anatomy. A suture anchor was used to repair the LCL complex. This is the same case as Figures 7 and 8. 


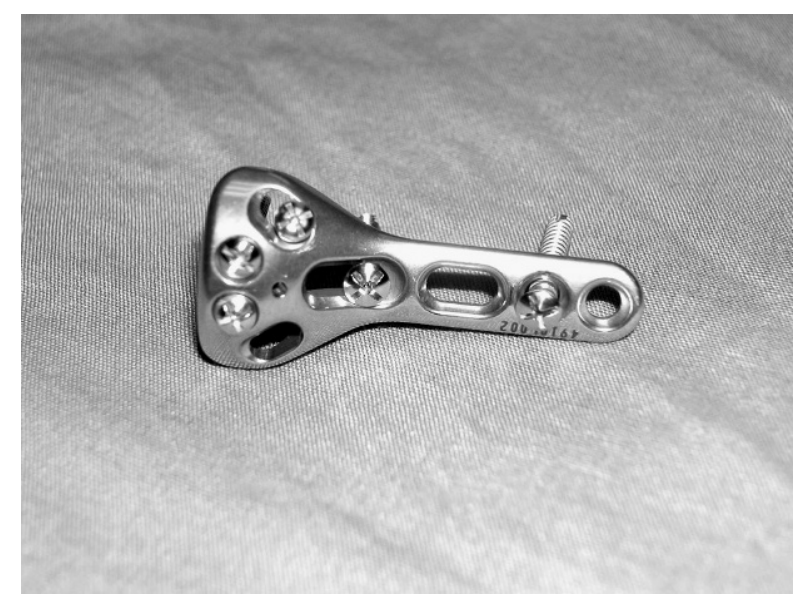

FIGURE 10. An example of a plate specifically designed for the radial head. There are multiple locked screws in the head, an elliptical hole for an interfragmentary or shaft screw, and the shape is precontoured (Wright Medical Technology, Arlington, Tenn).

revealed that ORIF provided acceptable results in all cases of noncomminuted Mason type II fractures and in 11 of 12 cases of type III fractures with 3 or fewer fragments. For fractures with greater comminution than this, the rates of hardware failure and nonunion were exceedingly high. It needs to be remembered that this study was performed before newer implants such as locking plates and plates dedicated for the radial head were available.

\section{COMPLICATIONS}

Complications after ORIF of the radial head can occur both early and late in the postoperative course. Early complications are seen with injury to the PIN and inappropriate hardware placement. Late complications include symptomatic hardware, nonunion, and elbow stiffness. Failure of fixation is noted with highly comminuted fractures, as demonstrate by Ring et al ${ }^{15}$ where 13 of 14 fractures involving the entire radial head and more than 3 articular fragments had fixation failure requiring revision surgery. This group also had 6 nonunions and 4 patients with less than 100 degrees of forearm rotation. Thus, only 1 patient with this difficult fracture pattern had an acceptable result.

\section{POSTOPERATIVE MANAGEMENT}

After radial head surgery, elbow motion and forearm rotation is necessary to prevent adhesions and stiffness. For these reasons, the goal of surgical repair of a radial head fracture should be to create a stable construct that allows early ROM. If the fixation is tenuous and would not permit early ROM, it is better to convert to a rigid implant arthroplasty.

If an LCL repair is performed, supination should be limited to 45 degrees to protect the lateral column and allow healing. Flexion increases elbow stability and can be permitted as tolerated, whereas extension should initially be blocked to 30 degrees. Extension can usually be increased by 10 degrees every 1 to 2 weeks under therapy guidance. Flexion and extension are done with the forearm in pronation, whereas rotation is performed at 90 degrees of elbow flexion. Another restriction that should be placed on postoperative motion is limitation of shoulder abduction in the presence of an LCL repair. By preventing abduction, the varus forces placed across the elbow, and ultimately the LCL, are reduced. Based on the quality of repair, a minimum of 6 weeks of limited abduction should allow the LCL to heal. ${ }^{26}$

\section{- FUTURE OF THE TECHNIQUE}

With the advent of locked-plate designs, smaller fixedangle devices are now being created to provide more
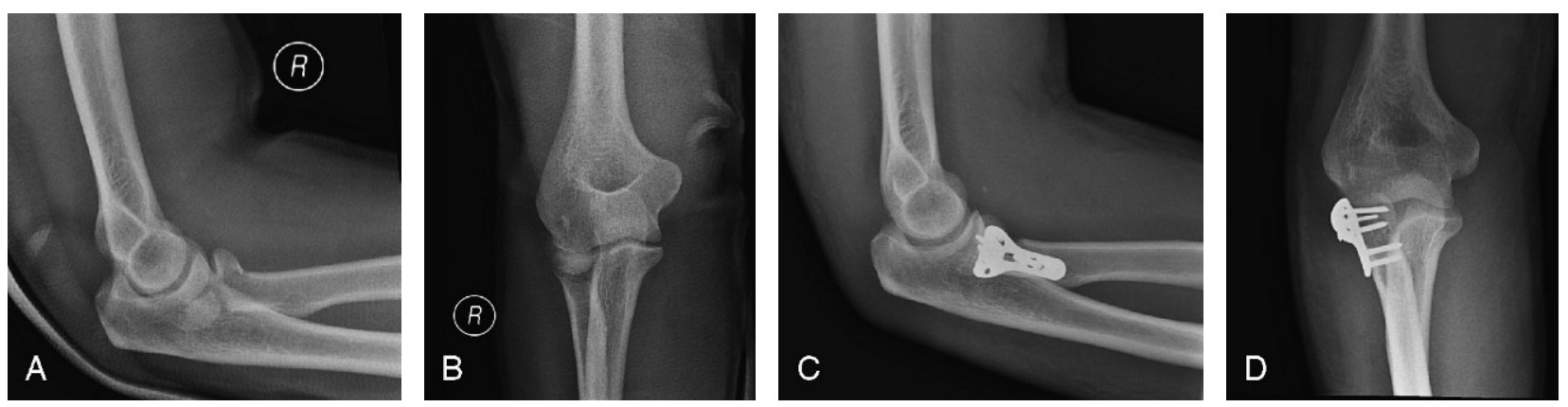

FIGURE 11. Lateral (A) and AP radiographs (B) showing a multifragmentary articular fracture. There is comminution in the neck portion of the fracture. Lateral $(C)$ and AP radiographs $(D)$ displaying stabilization of the fracture with a radial head plate. Because of the normal angulation of the radial head, a T plate will sit obliquely on the shaft when the radial head is properly reduced. 
rigid fixation for these fractures. Additionally, plates specifically designed for the radial head with multiple locked screws into the head are now available (Figs. 10 and 11). These small locking plates provide more secure fixation and may permit earlier ROM and could result in improved healing rates. Bioabsorbable implants have shown promise in other areas of fixation and may be beneficial for the radial head. These are attractive because they don't need to be removed and minimize potential impingement. ${ }^{27-29}$ Arthroscopically assisted fixation has shown promising results, and its indications should expand as the equipment and surgeons' experience improve. ${ }^{30,31}$

\section{REFERENCES}

1. Hall JA, McKee M. Posterolateral rotatory instability of the elbow following radial head resection. $J$ Bone Joint Surg Am. 2005;87:1571-1579.

2. Geel CW, Palmer A. Radial head fractures and their effect on the distal radioulnar joint. A rationale for treatment. Clin Orthop Relat Res. 1992:79-84.

3. Ikeda M, Sugiyama K, Kang C, et al. Comminuted fractures of the radial head: comparison of resection and internal fixation. Surgical technique. J Bone Joint Surg Am. 2006;88(suppl 1, pt 1):11-23.

4. Boulas HJ, Morrey BF. Biomechanical evaluation of the elbow following radial head fracture. Comparison of open reduction and internal fixation vs. excision, silastic replacement, and non-operative management. Chir Main. 1998; 17:314-320.

5. Hotchkiss RN. Displaced fractures of the radial head: internal fixation or excision? J Am Acad Orthop Surg. 1997;5:1-10.

6. Ikeda M, Sugiyama K, Kang C, et al. Comminuted fractures of the radial head. Comparison of resection and internal fixation. J Bone Joint Surg Am. 2005;87: 76-84.

7. Furry KL, Clinkscales CM. Comminuted fractures of the radial head. Arthroplasty versus internal fixation. Clin Orthop Relat Res. 1998;353:40-52.

8. Khalfayan E, Culp R, Alexander A. Mason type II radial head fractures: operative versus nonoperative treatment. J Orthop Trauma. 1992;6:283-289.

9. Beingessner DM, Dunning CE, Stacpoole RA, et al. The effect of radial head excision and arthroplasty on elbow kinematics and stability. J Bone Joint Surg Am. 2004;86: $1730-1739$.

10. Mason M. Some observations on fractures of the head of the radius with a review of one hundred cases. Br J Surg. 1959;42:123-132.

11. Ring D. In: Bucholz RWH, James D, Court-Brown C, et al, eds. Fractures and Dislocations of the Elbow. Rockwood and Green's Fractures in Adults, Vol. 1, 3rd ed. Philadelphia: Lippincott Williams \& Wilkins; 2005:1011-1020.

12. Capo JT, Riaszadeh K, Swan KG, et al. The accuracy of plain radiographs in assessing fractures of the radial head. Paper presented at: AAOS Annual Meeting; 2005; Washington, DC.

13. Akesson T, Herbertsson P, Josefsson P, et al. Primary nonoperative treatment of moderately displaced two-part fractures of the radial head. J Bone Joint Surg Am. 2006; 88:1909-1914.

14. Ikeda M, Oka Y. Function after early radial head resection for fracture: a retrospective evaluation of 15 patients followed for 3-18 years. Acta Orthop Scand. 2000;71: 191-194.

15. Ring D, Quintero J, Jupiter J. Open reduction and internal fixation of fractures of the radial head. J Bone Joint Surg Am. 2002;84:1811-1815.

16. Tan V, Daluiski A, Capo J, et al. Hinged elbow external fixators: indications and uses. J Am Acad Orthop Surg. 2005; 13:503-514.

17. Diliberti T, Botte MJ, Abrams RA. Anatomical considerations regarding the posterior interosseous nerve during posterolateral approaches to the proximal part of the radius. J Bone Joint Surg Am. 2000;82:809-813.

18. Strauch RJ, Rosenwasser M, Glazer P. Surgical exposure of the dorsal proximal third of the radius: how vulnerable is the posterior interosseous nerve? J Shoulder Elbow Surg. 1996;5:342-346.

19. Hoppenfeld S, deBoer P. The Elbow. Surgical Exposures in Orthopaedics, The Anatomic Approach, 3rd ed. Philadelphia: Lippincott Williams \& Wilkins; 2003.

20. Cohen MS, Hastings H II. Post-traumatic contracture of the elbow. Operative release using a lateral collateral ligament sparing approach. J Bone Joint Surg Br. 1998; 80:805-812.

21. Witt JD, Kamineni S. The posterior interosseous nerve and the posterolateral approach to the proximal radius. J Bone Joint Surg Br. 1998;80:240-242.

22. Caputo AE, Mazzocca A, Santoro V. The nonarticulating portion of the radial head: anatomic and clinical correlations for internal fixation. J Hand Surg. 1998;23:1082-1090.

23. Patterson JD, Jones CK, Glisson RR, et al. Stiffness of simulated radial neck fractures fixed with 4 different devices. J Shoulder Elbow Surg. 2001;10:57-61.

24. Capo JT, Asghar J, Sabatino C. Biomechanical stability of fixation constructs for ORIF of radial head fractures. Paper presented at: Annual Meeting of the American Society for Surgery of the Hand; 2003.

25. King G, Evans D, Kellam J. Open reduction and internal fixation of radial head fractures. J Orthop Trauma. 1991; 5:21-28.

26. Ring D. Radial head fractures: open reduction internal fixation. In: Wiss DA, ed. Master Techniques in Orthopaedic 
Surgery, Fractures, 2nd ed. Philadelphia: Lippincott Williams \& Wilkins; 2006:121-141.

27. Claes LE, Ignatius AA, Rehm KE, et al. New bioresorbable pin for the reduction of small bony fragments: design, mechanical properties and in vitro degradation. Biomaterials. 1996;17:1621-1626.

28. Pelto K, Hirvensalo E, Bostman O, et al. Treatment of radial head fractures with absorbable polyglycolide pins: a study on the security of the fixation in 38 cases. J Orthop Trauma. 1994;8:94-98.
29. Hirvensalo EO, Bostman B, Rokkanen P. Absorbable polyglycolide pins in fixation of displaced fractures of the radial head. Arch Orthop Trauma Surg. 1990;109: 258-261.

30. Rolla PR, Surace MF, Bini A, et al. Arthroscopic treatment of fractures of the radial head. Arthroscopy. 2006;22:231-236.

31. Dawson FA, Inostroza F. Arthroscopic reduction and percutaneous fixation of a radial neck fracture in a child. Arthroscopy. 2004;20(suppl 2):90-93. 